\title{
Urodinâmica Convencional versus Cistometria Simplificada para Caracterização da Incontinência Urinária Feminina
}

\author{
Conventional Urodynamics versus Simplified Cystometry for Characterization \\ of Female Urinary Incontinence
}

Paulo Roberto Kawano, João Luiz Amaro, Fábio Scarpa e Silva, Aparecido Donizete Agostinho, Hamilto Akihissa Yamamoto, José Carlos Souza Trindade

\section{RESUM0}

Objetivo: avaliar a concordância dos diferentes parâmetros urodinâmicos comparados à cistometria simplificada, permitindo uma diminuição na relação custo-benefício no diagnóstico da incontinência urinária de esforço (IUE) na mulher.

Métodos: foram coletadas e avaliadas retrospectivamente as informações contidas dos prontuários de trinta pacientes acompanhadas, no periodo de janeiro de 2000 a março de 2001. Todas foram submetidas a exame fisico geral e ginecológico. O estudo urodinâmico foi realizado pela técnica convencional, utilizando-se aparelho Dynograph Recorder R-611. A cistometria simplificada foi realizada com auxílio de um equipo em " $Y$ " de PVC (pressão venosa central), conectado a um sonda de Foley $14 \mathrm{~F}$, que permitia tanto a infusão de soro fisiológico como a captação da pressão intra-vesical. Foram analisados os parâmetros: volume residual, capacidade vesical, complacência, presença de contrações involuntárias do detrusor e perdas urinárias aos esforços. Para determinação da proporção de concordância entre os métodos foram utilizados o teste de concordância de Pearson e o teste de Wilcoxon, para amostras relacionadas.

Resultados: a média de idade foi de 50 anos, com extremos variando de 28 a 70 anos. $O$ indice de concordância entre os estudos, na demonstração das perdas urinárias aos esforços, foi de $67 \%$. Para a detecção das contrações involuntárias do detrusor, a proporção de concordância foi de 90\%. A média do volume residual encontrado na cistometria simplificada foi de 16,8 ml contra $2 \mathrm{ml}$ da urodinâmica convencional, com diferença significativa ( $p<$ 0,01 ). A média de capacidade vesical máxima no estudo urodinâmico foi de 440,5 ml, enquanto que, na cistometria simplificada, foi de $387 \mathrm{ml}(p<0,05)$. A complacência vesical foi, em média, significativamente maior na cistometria simplificada $\left(43,0 \mathrm{ml} / \mathrm{cmH}_{2} \mathrm{O}\right)$ quando comparada ao estudo urodinâmico (31,5 ml/ $\left.\mathrm{cmH}_{2} \mathrm{O}\right)$, com $p<0,01$.

Conclusão: avaliações preliminares sugerem que a propedêutica uroginecológica associada à cistometria simplificada é uma opção a ser considerada na avaliação clínica e pré-operatória de pacientes com IUE em substituição à urodinâmica convencional, particularmente onde esta última não se encontra disponível. A cistometria simplificada é um exame acessível que é capaz de detectar contrações involuntárias do detrusor, assim como identificar perdas urinárias com relativa sensibilidade, proporcionando ao examinador noções fidedignas do comportamento vesical.

PALAVRAS-CHAVE: Cistometria simplificada. Estudo urodinâmico. Incontinência urinária.

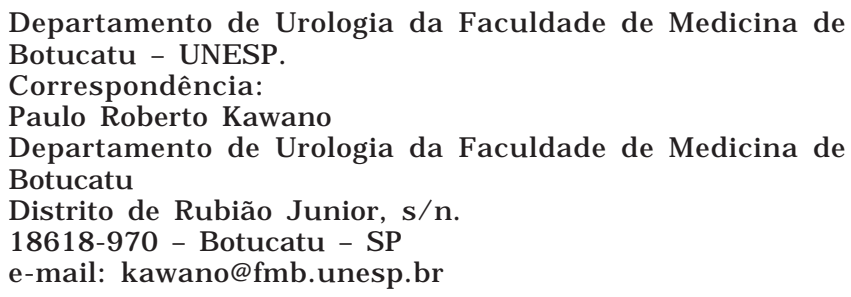

\section{Introdução}

A incontinência urinária de esforço (IUE) na mulher determina significativas mudanças na sua qualidade de vida, sendo responsável pelo aumento da incidência de distúrbios psicossociais e se- 
xuais como perda da auto-estima e depressão ${ }^{1,2}$. O sucesso do tratamento da incontinência urinária depende do diagnóstico correto, objetivando-se a correção da disfunção ou alteração que causa a perda de urina. A história clínica e o exame físico completo são fundamentais na avaliação desses casos. Pacientes com imperiosidade miccional, urge-incontinência, enurese noturna ou que foram submetidas a tratamento cirúrgico prévio devem ter sua avaliação complementada com estudo urodinâmico ${ }^{3,4}$. A importância do estudo urodinâmico nestas pacientes está em orientar o diagnóstico, auxiliar na escolha da forma de tratamento e fornecer informações complementares para o seguimento pós-cirúrgico ${ }^{5-9}$.

Embora de grande acurácia, trata-se de um exame específico que necessita de aparato tecnológico apropriado e de profissionais capazes de executá-lo e de interpretar de forma correta seus resultados. Além disso, diversos estudos têm demonstrado a associação do exame urodinâmico com taxas de morbidade e complicações diversas ${ }^{10}$, com incidência de infecções pós-realização do exame variando de 2 a $30 \%{ }^{1}$. Gonnermann et al. ${ }^{5}$ reportaram boa tolerabilidade ao exame, porém um terço das pacientes manifestaram desejo de não serem submetidas novamente a tal procedimento ${ }^{11,12}$.

Atualmente têm sido propostas diversas técnicas simplificadas para realização do estudo urodinâmico capazes de reduzir sua complexidade e eliminar a necessidade do emprego de alta tecnologia. Uma vez constatada sua reprodutibilidade e confiabilidade, tais modalidades permitiriam uma redução do custo final do exame, sem prejuízo na qualidade de seus resultados. Pushkar et al. ${ }^{12}$ avaliaram o potencial diagnóstico de testes urodinâmicos combinados em diferentes formas de incontinência urinária, demonstrando que a cistometria foi mais eficiente no diagnóstico da instabilidade do detrusor ${ }^{13,14}$.

Avaliações preliminares sugerem que a propedêutica uroginecológica, associada à cistometria, é opção a ser considerada na avaliação clínica e pré-operatória de pacientes com IUE em substituição à urodinâmica convencional, particularmente onde esta última não se encontra disponivel. Trata-se de exame acessivel e de considerável sensibilidade para a detecção de contrações vesicais involuntárias, além de proporcionar ao examinador noções fidedignas da complacência vesical e da atividade vesical ${ }^{6,7}$.

Este trabalho tem por objetivo avaliar a concordância dos diferentes parâmetros urodinâmicos em relação à cistometria simplificada, bem como determinar suas limitações quando comparada à urodinâmica convencional.

\section{Pacientes e Métodos}

Foram coletadas e avaliadas retrospectivamente as informações contidas dos prontuários de trinta pacientes acompanhadas no Serviço de Uroginecologia do Hospital das Clínicas da Faculdade de Medicina da Botucatu - UNESP, no período de janeiro de 2000 a março de 2001. Tais pacientes eram portadoras de incontinência urinária de esforço, caracterizada por perdas urinárias aos minimos esforços como tossir, espirrar ou durante atividades físicas leves como subir escadas ou caminhar depressa. Tais queixas foram confirmadas pela demonstração objetiva da perda urinária durante o exame físico, solicitando-se à paciente que realizasse a manobra de Valsalva. Todas as pacientes foram submetidas a exame físico e ginecológico completos, estudos urodinâmicos e cistométricos. Nenhuma delas havia sido submetida à cirurgia prévia para tratamento de incontinência urinária.

O estudo urodinâmico foi realizado pela técnica habitual, com a paciente em decúbio dorsal, empregando-se aparelho Dynograph Recorder R611. Inicialmente foi realizada fluxometria obtendo-se assim o fluxo urinário máximo (Qmáx). Após a anti-sepsia da genitália, introduziu-se sonda vesical $\mathrm{n}^{\mathrm{o}} 10 \mathrm{Fr}$ de duas vias, medindo-se então o resíduo pós-miccional. Um balão retal foi introduzido com o objetivo de medir a pressão abdominal (Pabd). A seguir, iniciou-se a infusão lenta de solução fisiológica intravesical, obtendo-se assim a medida da pressão máxima do detrusor (Pdet.máx.), calculada pela diferença entre a pressão intravesical (Pves) e abdominal (Pabd). Considerou-se hiperatividade vesical quando da observação de contrações involuntárias do detrusor durante a fase de enchimento vesical, espontânea ou provocada ${ }^{15,16}$.

A pressão de perda sob esforço (PPE) foi obtida com a paciente em posição deitada, com capacidade vesical máxima. Para isso, realizou-se manobra de esforço observando-se a pressão abdominal mínima necessária para que ocorresse a perda de urina na ausência de contração involuntária do detrusor.

A complacência vesical foi calculada utilizando a seguinte fórmula: $\mathrm{C}=\mathrm{Vf}-\mathrm{Vi} / \mathrm{Pf}$ - $\mathrm{Pi}$, onde: $\mathrm{C}=$ complacência vesical $\left(\mathrm{mL} / \mathrm{cmH}_{2} \mathrm{O}\right) ; \Delta \mathrm{v}=$ volume final (Vf) - volume inicial (Vi); $\Delta \mathrm{p}=$ pressão final (Pf) - pressão inicial (Pi).

A cistometria simplificada foi realizada após exame ginecológico com a paciente em decúbito dorsal em posição de litotomia, procedendo-se inicialmente a sondagem vesical com sonda tipo 
Foley 14 F. Utilizando-se um equipo em "Y" de PVC graduado (equipo de medida da pressão venosa central) conectado a um reservatório de solução salina, foi possivel o registro da pressão intravesical e a infusão de solução fisiológica. Tais mensurações foram efetuadas a cada $50 \mathrm{~mL}$, até que a paciente referisse sensação de plenitude vesical, com taxa de infusão aproximada de $10 \mathrm{~mL} / \mathrm{minuto}$. A pressão "zero" foi obtida no nível da sínfise púbica com a bexiga completamente vazia. A presença de contrações não inibidas foi identificada pela oscilação maior que $5 \mathrm{~mm}$ do nível da solução salina no interior do equipo e/ou pelo refluxo do líquido para o reservatório, no caso de contrações vesicais mais intensas. $\mathrm{O}$ volume residual foi obtido pela simples sondagem vesical antes da realização do exame, após a paciente ter esvaziado a bexiga A presença de perda urinária aos esforços foi avaliada solicitandose à paciente que realizasse manobras de Valsalva até atingirmos a capacidade vesical máxima.

Os parâmetros analisados em ambas as situações foram: volume residual, capacidade vesical, complacência, presença de contrações involuntárias do detrusor e perda urinária aos esforços. Para determinação da proporção de concordância entre os métodos foram utilizados o teste de concordância de Pearson e o teste de Wilcoxon, para amostras relacionadas, sendo considerados significativos quando $\mathrm{p}<0,05$.

\section{Resultados}

O índice de concordância entre cistometria simplificada e o estudo urodinâmico foi de $67 \%$ na detecção de perdas urinárias durante esforço ${ }^{17}$. Nas contrações involuntárias do detrusor, a proporção de concordância foi de $90 \%$ entre os diferentes métodos (Tabela 1).

Tabela 1 - Proporção de concordância entre os métodos de avaliação da incontinência urinária feminina, e seus respectivos limites de confiança (teste de concordância de Pearson).

\begin{tabular}{lccc}
\hline Variável & $\begin{array}{c}\text { Proporção de } \\
\text { concordância }\end{array}$ & $\begin{array}{c}\text { Limite } \\
\text { inferior }\end{array}$ & $\begin{array}{c}\text { Limite } \\
\text { superior }\end{array}$ \\
\hline Contração involuntária & 0,9 & 0,8 & 1,0 \\
Perda urinária & 0,67 & 0,5 & 0,84 \\
\hline
\end{tabular}

A média do volume residual na cistometria simplificada $(16,8 \mathrm{~mL})$ foi significativamente maior quando comparada à urodinâmica convencional $(2 \mathrm{~mL})(\mathrm{p}<0,01)$.

A média da capacidade vesical máxima no estudo urodinâmico foi de $440,5 \mathrm{~mL}$, ao passo que, na cistometria simplificada, foi de $387 \mathrm{~mL}$, com diferença significativa $(\mathrm{p}<0,05)$. A média da complacência vesical foi significativamente maior na cistometria simplificada $\left(43,0 \mathrm{~mL} / \mathrm{cmH}_{2} \mathrm{O}\right)$ quando comparada ao estudo urodinâmico $(31,5 \mathrm{~mL} /$ $\mathrm{cmH}_{2} \mathrm{O}$ ), com $\mathrm{p}<0,01$ (Tabela 2).

Tabela 2 - Medianas dos diferentes parâmetros e resultado do teste estatístico (teste de concordância de Pearson/Wilcoxon) para comparação entre os métodos de avaliação da incontinência urinária feminina.

\begin{tabular}{lccc}
\hline Variável & Método & & \\
& Cistometria simplificada & Urodinâmica convencional & \\
\hline Volume residual $(\mathrm{mL})$ & 5,0 & 0,0 & $\mathrm{p}<0,01$ \\
Capacidade vesical $(\mathrm{mL})$ & 387,0 & 440,5 & $\mathrm{p}<0,05$ \\
Complacência $\left(\mathrm{mL} / \mathrm{cmH}_{2} \mathrm{O}\right)$ & 43,0 & 31,5 & $\mathrm{p}<0,01$ \\
\hline
\end{tabular}

\section{Discussão}

Sob o aspecto emocional, o estudo urodinâmico pode ser exame agressivo e constrangedor, pois a paciente é submetida à introdução de cateteres no reto e na bexiga, expondo suas sensações durante o exame; é obrigada a urinar em um ambiente completamente estranho e, não raramente, pode apresentar perda urinária diante do médico ou da enfermeira ${ }^{6}$. Embora de grande acurácia, trata-se de exame específico e de considerável custo.
Há muito tempo têm sido propostas diversas técnicas simplificadas para realização do estudo urodinâmico capazes de reduzir sua complexidade e eliminar a necessidade do emprego de alta tecnologia. Dentre a opções disponíveis, a cistometria simplificada deve ser considerada, uma vez que tem se mostrado exame de fácil realização e de baixo custo, não sendo dependente de qualquer tipo de aparato eletrônico para sua execução. A cistometria foi primeiro descrita por Mosso e Pellacani ${ }^{18}$ e introduzida como método de rotina na investigação de distúrbios vesicais funcionais por Lewis, em $1939^{19}$. O emprego da 
cistometria permite a documentação de inúmeros parâmetros como a capacidade vesical, pressões vesicais em diferentes volumes, o volume pósmiccional e a presença de contrações involuntárias do detrusor ${ }^{20}$.

Embora de grande importância, as informações fornecidas pela complacência vesical devem ser avaliadas com cuidado, uma vez que estão sujeitas a interferência de inúmeros fatores inerentes a cada paciente e que podem distorcer seus resultados, independentemente do método utilizado ${ }^{20}$.

No presente estudo, a maior complacência vesical observada no estudo urodinâmico é explicada pelos maiores valores da capacidade vesical encontrados quando comparados à cistometria. Este fato pode ter ocorrido, possivelmente, pela variação individual da sensibilidade vesical, uma vez que a infusão da solução fisiológica só era suspensa quando a paciente referisse desejo miccional imperioso ou dor. Sorensen et al. ${ }^{21}$, em 1984, encontraram variações do volume tanto na primeira sensação vesical, quanto na capacidade vesical máxima em pacientes submetidos a quatro cistometrias realizadas em diferentes momentos, variando-se apenas a taxa de infusão ou a posição da paciente. Alterações dos parâmetros sensoriais vesicais podem acontecer por variações individuais e, entre outros motivos, pelo fato de que contrações involuntárias do detrusor são mais facilmente deflagradas com a paciente em posição ortostática que em decúbito dorsal $^{22}$. Assim sendo, outro fator contributivo, na opinião dos autores, pode ter sido a posição da paciente. Durante a realização do estudo urodinâmico, a paciente permanecia em decúbito dorsal horizontal, ao passo que, na cistometria simplificada, a posição de litotomia na mesa ginecológica provavelmente causava maior desconforto e, conseqüentemente, diminuição da capacidade vesical máxima. Sorensen, em $1988^{23}$, realizou duas cistometrias consecutivas em 12 pacientes menopausadas, sendo a primeira em posição supina e a segunda com a paciente sentada, nas quais os autores observaram um incremento tanto dos valores pressóricos como da capacidade vesical entre os diferentes grupos ${ }^{23}$.

A discordância dos resultados obtidos nas medidas do volume residual pode ser explicada pela falta de padronização, seja nas instruções fornecidas à paciente, seja na variação do tempo decorrido entre o final do exame e a passagem da sonda para medida do volume residual pósmiccional.

De acordo com Wall et al. ${ }^{24}$, a demonstração de perdas urinárias na incontinência urinária de esforço genuína durante a cistometria simples tem sensibilidade de $88,1 \%$ e especificidade de $77,1 \%$, com valor preditivo positivo de $82 \%$. No presente estudo, a sensibilidade da cistometria simplificada, na detecção das perdas urinárias, foi de 67\% quando comparada à urodinâmica convencional. van Waalwijk van Doorn ${ }^{25}$, utilizando a cistouretrometria, observou baixa sensibilidade no diagnóstico de incontinência urinária de esforço pura e mista, em que apenas 164 dos 348 pacientes (47\%) apresentaram perda durante o exame, sendo a sensibilidade de 53,32\% para incontinência de esforço pura e $13 \%$ na incontinência mista. O mesmo autor, em estudo envolvendo 80 pacientes apresentando incontinência urinária de esforço pura ou associada a urge-incontinência, utilizando apenas a cistometria simples, foi capaz de demonstrar perda urinária em apenas 31 pacientes, cerca de $39 \%{ }^{26}$.

Resultados conflitantes têm sido encontrados, particularmente no que se refere a parâmetros sensoriais obtidos durante a cistometria. Não há muitos dados sobre a confiabilidade desses resultados, uma vez que revisões da literatura sobre a cistometria e seus achados estão baseadas em trabalhos publicados entre 1987 e 1993, com apenas cinco artigos encontrados. A reprodutibilidade de um ou mais parâmetros sensoriais foi observada em alguns, mas não em todos os artigos ${ }^{27}$. Tal problema ocorre principalmente em pacientes que apresentam urge-incontinência, nas quais pode ser difícil determinar se a urgência antecede ou se é responsável pela perda urinária ${ }^{20}$.

Tanto a cistometria simplificada quanto o estudo urodinâmico avaliam apenas uma pequena janela da atividade vesical por cerca de 20 a 30 minutos e as condições no laboratório durante o exame podem não reproduzir as situações do dia a dia da paciente ${ }^{28}$. Trabalhos recentes utilizando estudo urodinâmico ambulatorial, com monitorização da atividade vesical por várias horas, têm demonstrado que a maioria das pacientes com urge-incontinência apresentam evidências de graus variáveis de hiperatividade do detrusor ${ }^{29-31}$.

A cistometria simplificada foi comparada ao estudo urodinâmico por Ouslander et al. ${ }^{32}$, sendo que, das 171 pacientes geriátricas incontinentes avaliados pelo autor, 110 tinham instabilidade do detrusor ou hiper-reflexia ao estudo urodinâmico. A cistometria simples teve sensibilidade de $75 \%$ e especificidade de $79 \%$, com valor preditivo positivo de $85 \%$, na detecção destas alterações no mesmo grupo estudado.

A medida da pressão vesical obtida durante a cistometria simples pode ser útil na detecção de contrações involuntárias do detrusor, uma vez que 
estas podem levar a paciente a apresentar perda urinária. No entanto, como a bexiga é um órgão abdominal, alterações na pressão intra-abdominal podem ser interpretadas erroneamente como contrações do detrusor, se apenas as flutuações pressóricas na bexiga forem o único fator avaliado durante o exame ${ }^{24}$. De acordo com Wall et al. ${ }^{24}$, a cistometria simples tem sensibilidade de 64\%, mas especificidade de $86,8 \%$, na detecção de instabilidade do detrusor. No presente estudo, a concordância entre a cistometria simplificada e o estudo urodinâmico foi de $90 \%$ para a detecção das contrações involuntárias do detrusor.

Dentre as desvantagens apresentadas pela cistometria, destaca-se a impossibilidade da obtenção da pressão do detrusor (Pdet) durante a fase miccional. Este fato pode ser de grande importância no diagnóstico diferencial entre falência detrusora e obstrução infravesical, não permitindo a distinção das pacientes que apresentarão dificuldade à micção após correção cirúrgica da IUE. Wall et al. ${ }^{24}$ concluíram que, se a paciente possui causa única para sua incontinência e se os dados oferecidos por um estudo simplificado da bexiga forem condizentes com suas queixas, o tratamento precoce pode ser realizado. Algumas pacientes podem necessitar avaliação diagnóstica mais detalhada se o estudo for inconclusivo, suspeito, ou apontar para um caso de incontinência mista. Estudo urodinâmico complementar pode ser útil para confirmar diagnósticos não bem esclarecidos, classificar subtipos de incontinência urinária e proporcionar dados adicionais sobre outros aspectos da função vesical ${ }^{20}$. Entretanto, nas pacientes sem alterações neurológicas ou cirurgias prévias, a cistometria simplificada pode fornecer dados importantes, seja na identificação de contrações involuntárias do detrusor seja na determinação das perdas urinárias, em que, de acordo com o presente estudo, este exame alcançou concordância com o estudo urodinâmico de 90 e 67\%, respectivamente. Esta forma simplificada de estudo da dinâmica vesical pode fornecer ao médico informações importantes e necessárias à confirmação do diagnóstico e à avaliação pré-operatória de pacientes portadoras de IUE.

\section{ABSTRACT}

Objective: to assess the concordance of different urodynamic parameters with simplified cystometry, thus improving the cost-benefit relationship for stress urinary incontinence (SUI) diagnosis in woman.
Methods: we evaluated retrospectively the medical records of thirty patients treated, from January 2000 to March 2001. All patients had been submitted to physical and gynecological examinations. A conventional urodynamic study had been made using a Dynograph R-611 recorder. Simplified cystometry had used a saline tube with " $Y$ " connector, connected to a Foley $14 \mathrm{Fr}$ catheter, which allowed measurement of intravesical pressure at the same time as physiological saline infusion. The following parameters were analyzed: residual volume, bladder capacity, complacency, involuntary detrusor contractions, and abdominal leak-point pressure. The Pearson test of agreement and the Wilcoxon signed rank test were used to verify the concordance between related samples, with $p<0,05$.

Results: the average age was 50 years old (28-70). Concordance between studies for stress urinary losses was $67 \%$, and for detrusor involuntary contractions, $90 \%$. The average residual volume was significantly different: by simplified cystometry it was $16.8 \mathrm{ml}$ versus $2 \mathrm{ml}$ by conventional urodynamics $(p<0.01)$. The average maximum vesical capacity by urodynamic study was $440.5 \mathrm{ml}$, and by simplified cystometry, $387 \mathrm{ml}(p<0.05)$. Vesical complacency was on average, significantly larger in simplified cystometry $\left(43.0 \mathrm{ml} / \mathrm{cmH}_{2} \mathrm{O}\right)$ than in the urodynamic study $(31.5 \mathrm{ml}$ $\left.\mathrm{CmH}_{2} \mathrm{O}\right)$, with $p<0.01$.

Conclusion: preliminary evaluations suggest that the urogynecologic propedeutic associated with cystometry is an option to be considered in the clinical and preoperative assessment of patients with SUI instead of conventional urodynamics, particularly when the latter is not available. Simplified cystometry is an accessible exam that grants comparable results for the detection of involuntary detrusor contractions and for the identification of urinary loss, providing the examiner with trustworthy data on vesical behavior.

KEYWORDS: Simplified cystometry. Urodynamic study. Urinary incontinence.

\section{Referências}

1. Gorton E, Staton S. Women's attitudes to urodynamics: a questionnaire survey. Br J Obstet Gynaecol 1999; 106:851-6.

2. Hafner RJ, Stanton SL, Guy J. A psychiatric study of women with urgency and urgency incontinence. Br J Urol 1977; 49:211-4.

3. Sander P, Mouritsen L, Andersen JT, FischerRasmussen W. Evaluation of a simple nonsurgical concept for management of urinary incontinence (minimal care) in an open- access, interdisciplinary incontinence clinic. Neurourol Urodyn 2000; 19:9-17.

4. D’Ancona CAL, Netto Júnior NR. Aplicações clínicas da urodinâmica. $1^{\mathrm{a}}$ ed. Campinas: Cartigraf; 1995. 
5. Gonnermann O, Hofner K, Krah H, Grunewald V, Jonas U. Urodynamics - assessment of morbidity. Neurourol Urodyn 1996; 15:305-6.

6. Rubstein I. Incontinência urinária na mulher. $1^{\mathrm{a}}$ ed. São Paulo: Atheneu; 2001.

7. Klingler HC, Madersbacher S, Djavan B, Schaltzl G, Marberger M, Schmidbauer CP. Morbidity of evaluation of the lower urinary tract with transurethral multichannel pressure-flow studies. J Urol 1998; 159:191-4.

8. Schafer W, Abrams P, Liao L, et al. Good urodynamic practices: uroflowmetry, filling cystometry, and pressure-flow studies. Neurourol Urodyn 2002; 21:261-74.

9. McGuire EJ, Cespedes RD, O'Connel HE. Leak-point pressures. Urol Clin North Am 1996; 23:253-62.

10.Bennes C, Manning J. Patient evaluation of urodynamic investigations. Neurourol Urodyn 1997; 16:509-10.

11.Teba del Pino F, Virseda Chamorro M, Salinas Casado J, Arredondo Martinez F, Fernandez Lao A, Fernandez Lucas C. Female urinary incontinence: clinical-urodynamic correlation. Arch Esp Urol 1999; 52:237-42.

12.Pushkar DIu, Loran OB, Gumin LM, D'iakin VV. The diagnostic value of combined urodynamic study in different forms of urinary incontinence in women. Urol Nefrol (Mosk) 1996; (4):21-5.

13. Vandeberg C. Female incontinence: critical analysis of diagnostic procedures. Acta Urol Belg 1995; 63:35-6.

14.Fink D, Perucchini D, Schaer GN, Haller U. The role of frequency-volume chart in the differential diagnostic of female incontinence. Acta Obstet Gynecol Scand 1999; 78:254-7.

15.Elbadawi A, Yalla SV, Resnick NM. Structural basis of geriatric voiding dysfunction. I. Methods of a prospective ultrastructural/urodynamic study and overview of the findings. J Urol 1993;150:1650-6.

16.Fank C, Brooks P. Practice tips. Bedside cystometry. Simple diagnostic skill for family physicians. Can Fam Physician 2000; 46:559-60.

17.Bland JM, Altman DG. Statistical methods for assessing agreement between two methods of clinical measurement. Lancet 1986; 1:307-10.

18. Mosso O, Pellacani P. Sur les fonctions sur la vessie. Arch Ital Biol 1882; 1:97-128.
19.Lewis LG. A new clinical recording cystometer. J Urol 1939; 41:638-45.

20.Kulseng-Hanssen S. Reliability and validity of stationary cystometry, stationary cystourethrometry and ambulatory cysto-urethrovaginometry. Acta Obstet Gynecol Scand Suppl 1997; 166:33-8.

21.Sorensen SS, Nielsen JB, Norgaard JP, Knudsen LM, Djurhuus JC. Changes in bladder volumes with repetition of water cystometry. Urol Res 1984; 12:205-8.

22.Awad SA, McGinnis RH. Factors that influence the incidence of detrusor instability in women. J Urol 1983; 130:114-5.

23.Sorensen S. Urodynamic investigations and their reproducibility in healthy postmenopausal females. Scand J Urol Nephrol Suppl 1988; 114:42-7.

24.Wall LL, Wiskind AK, Taylor PA. Simple bladder filling with a cough stress test compared with subtracted cystometry for the diagnosis of urinary incontinence. Am J Obstet Gynecol 1994; 171:1472-9.

25.van Waalwijk van Doorn ES. Ambulatory urodynamics: from physiological research to daily practice [thesis]. Amsterdam: Univ. of Maastricht; 1993.

26.van Waalwijk van Doorn ES, Janknegt RA. A telemetric ambulatory method to investigate the lower urinary tract function. Urodyn Neurourol Urodyn Continence 1993; 3:59-69.

27.Sorensen S, Gregersen H, Sorensen SM. Long term reproducibility of urodynamic investigations in healthy fertile females. Scand J Urol Nephrol Suppl $1988 ; 114: 35-41$.

28. McGuire E. Bladder instability and stressincontinence. Neurourol Urodyn 1988; 7:563-7.

29.James D. Continuous monitoring. Urol Clin North Am 1979; 6:125-35.

30.Bhatia NN, Bradley WE, Haldeman S. Urodynamics: continuous monitoring. J Urol 1982; 128:963-8.

31.van Waalwijk van Doorn ES, Remmers A, Janknegt RA. Extramural ambulatory urodynamic monitoring during natural filling and normal daily activities: evaluation of 100 patients. J Urol 1991; 146:124-31.

32. Ouslander J, Leach G, Abelson S, Staskin D, Blaustein J, Raz S. Simple versus multichannel cystometry in the evaluation of bladder function in an incontinent geriatric population. J Urol 1988; 140:1482-6. 